\title{
Viewpoint Adaptation for Person Detection
}

\author{
Patrick Wang ${ }^{1,2}$, Kenneth Morton ${ }^{2}$, Peter Torrione ${ }^{2}$, and Leslie Collins ${ }^{1}$ \\ 1 Duke University \\ 2 CoVar Applied Technologies \\ \{patrick.wang, leslie.collins\}@duke.edu \\ $\{$ kenny, pete\}@covar.com
}

\begin{abstract}
An object detector performs suboptimally when applied to image data taken from a viewpoint different from the one with which it was trained. In this paper, we present a viewpoint adaptation algorithm that allows a trained single-view person detector to be adapted to a new, distinct viewpoint. We first illustrate how a feature space transformation can be inferred from a known homography between the source and target viewpoints. Second, we show that a variety of trained classifiers can be modified to behave as if that transformation were applied to each testing instance. The proposed algorithm is evaluated on a new synthetic multi-view dataset as well as images from the PETS 2007 and CAVIAR datasets, yielding substantial performance improvements when adapting single-view person detectors to new viewpoints while increasing the detector frame rate. This work has the potential to improve person detection performance for cameras at non-standard viewpoints while simplifying data collection and feature extraction.
\end{abstract}

Keywords: viewpoint, domain, adaptation, perspective, projection, pedestrian, detection

\section{Introduction}

The automated detection of persons in images is an important task in a wide variety of applications: autonomous driving, robotics, surveillance, image annotation, and others. While the problem to be solved in each of these scenarios is fundamentally the same, each is associated with different expectations for the position and orientation of the camera.

Recent work, for example, has developed and refined algorithms for pedestrian detection, i.e. the detection of persons from a camera mounted on the hood of a car $[1,2]$. Due to this unique camera pose, these detectors typically view persons only one way: horizontally at about waist level. This viewpoint is reflected in the popular datasets used to evaluate pedestrian detection algorithms $[3,4]$. Pedestrian detectors will not perform optimally if applied to, for example, a mall security camera, which may see persons from a wide variety of relative viewpoints (see Figure 1). If one could leverage camera pose information along with the existing body of work in pedestrian detection, we could develop fast and accurate person detectors for cameras with arbitrary (known) poses. 


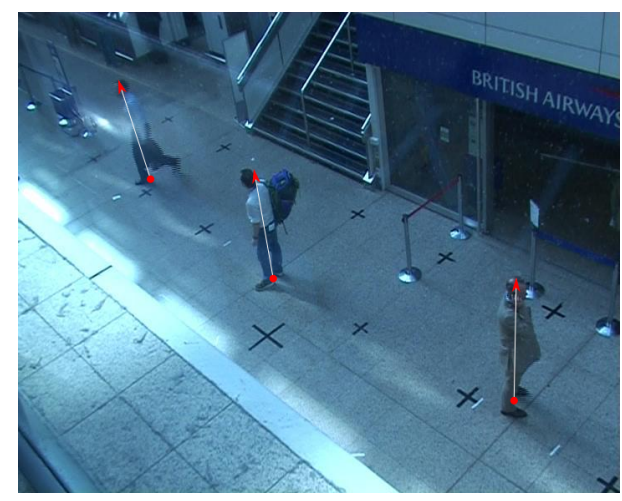

Fig. 1. Example surveillance camera image from the PETS 2007 dataset (camera 3) [5]. The size, orientation, and proportions of person images depend on their position and orientation relative to the camera. Line segments connecting the persons' heads and feet illustrate variations in orientation.

More generally, it is desirable to be able to adapt an object detector trained for one viewpoint to another distinct viewpoint. This is an instance of the "domain adaptation" problem, in which the conditions under which a detector is to be applied do not match the those under which the training data were collected [6]. "Domain," can refer to a variety of factors like viewpoint, lighting, and resolution.

The camera viewpoint is of particular interest because it can be defined entirely by a small number of geometric parameters which can be estimated from the visual scene. We will show that knowledge of the camera's viewpoint can be used to decouple the effects of viewpoint from other domain factors and to learn a feature space transformation between the source and target viewpoints. The feature transformation can then be used to adapt a trained object detector to the target viewpoint. This novel adaptation procedure enables improved detection across different viewpoints while requiring very little additional run-time computation. Furthermore, our approach does not require data from the target domain, in contrast with existing domain adaptation algorithms.

\section{Related Work}

Many domain adaptation algorithms begin with an arbitrary single- or multiview detector and select or re-weight instances so that they better reflect the distribution of instances from the viewpoint of interest $[16,17]$. Others explicitly learn a feature space transformation using data from the source and target domains [18]. These existing domain adaptation algorithms universally require training images from both the source and target domain and they often also require the corresponding labels from one or both domains. For many applications, however, it is impractical to manually collect new data and retrain the 
detector for every viewpoint that might be encountered during operation. For moving cameras or those with wide-angle lenses capturing a continuous range of relative perspectives, this will be impossible.

An assortment of methods have been designed to work around this viewpoint problem. Some object detectors instead extract features designed to be invariant to small geometric transformations of various types. For example, scale-invariant feature transforms (SIFT) [7] are partially invariant to changes in objects' scale. Histograms of oriented gradients (HOG) [3] are invariant to small local rotations. Such viewpoint-invariant local features are important to help normalize pose differences in deformable objects. They cannot, however, capture large-scale shape changes, like those illustrated in Figure 1.

For situations like this, many existing methods attempt to leverage powerful machine learning techniques to recognize objects from any viewpoint. These rely on having training data from all viewpoints of interest, and use robust classifiers to distinguish between the (very diverse) class of target instances and the class of background instances. The classification problem resulting from such "multiview" approaches may be much more complex than detecting persons from only a single viewpoint. Furthermore, it is usually difficult to collect training data sets containing a comprehensive range of perspectives.

Some approaches attempt to avoid these issues by using a 3D model along with images from a small number of "canonical" views to detect objects with arbitrary relative poses [8-10]. These do not take advantage of any known camera pose information, and can suffer from high runtime computational requirements.

For ease of computation, the assumption of a planar model is common in optical character recognition $[11,12]$ and has also been used in face recognition [13] and in some general object detection approaches [14]. Li et al. [15] apply this planar assumption to person detection, modelling persons as planes perpendicular to the ground, allowing them to warp candidate detection windows to match the trained classifier's viewpoint. An image warping procedure like this is illustrated in Figure 2. They show that significant performance improvements may be obtained with this method compared to using a single-view classifier without compensatory warping. However, image warping involves (at run-time) extracting features from each candidate detection window independently, which is impractical for real-time applications.

In contrast, we approach the problem by modifying the trained detector itself to fit the viewpoint in which it will be used. Other work in computer vision that transforms detection models focuses only on scale $[19,2]$.

\section{Viewpoint Adaptation}

Rather than applying a projective warping to each testing window and then computing features (as in [15]), we approximate this process by instead applying an appropriate linear transformation to the features themselves. For some classes of detectors, we can further improve computational efficiency by observ- 

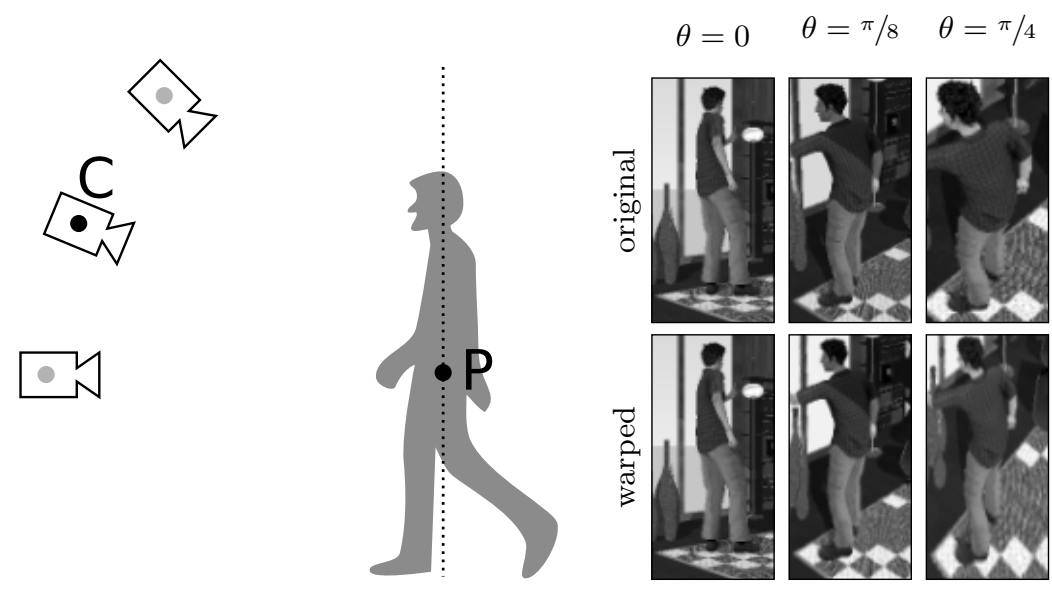

Fig. 2. Left, the person is assumed to be planar, lying on the plane indicated by the dotted line. Three viewpoints are considered, from cameras at elevations of $0, \pi / 8$, and $\pi / 4$ radians. Right, images from each elevation are warped to approximate 0 elevation (no transformation is necessary for the $0 \rightarrow 0$ case). The approximation degrades as elevation increases due to inaccuracies in the planar human model.

ing that this feature transformation is equivalent to a suitable transformation of the trained classifier parameters.

The classifier can thus be adapted to the target viewpoint, requiring nothing but a trained single-view classifier and a homography relating the source and target viewpoints. This approach has the benefit of needing only training data from a single viewpoint, so that extensive effort need not be spent acquiring diverse training images. In particular, no data is required from the specific viewpoint in which the classifier will be applied; it is sufficient to have knowledge of the camera's pose.

\subsection{Camera Homography}

If we define the camera's position $C$ and the person's position $P$, the person is assumed to be planar and perpendicular to the projection of the line segment $C P$ onto the ground plane. This plane is illustrated by the dotted line in Figure 2 . A point on this person plane is described using homogeneous coordinates by $\boldsymbol{u}_{p}=\left[u_{p}, v_{p}, 1\right]^{T}$. The corresponding point in image $i, \boldsymbol{u}_{i}=\left[u_{i}, v_{i}, 1\right]^{T}$, is related to the person-plane point by $\boldsymbol{u}_{i}=H_{i} \boldsymbol{u}_{p}$, where $H_{i}$ is a 3 -by-3 homography matrix. Then the relationship between points in images from two cameras with different viewpoints is given by $\boldsymbol{u}_{1}=H_{1} H_{2}^{-1} \boldsymbol{u}_{2}=H_{12} \boldsymbol{u}_{2}$. 


\section{$3.2 \quad$ Local Feature Re-mapping}

Many object detection algorithms can be defined in terms of a set of local image feature descriptors that are computed for each frame, and a sliding window classifier that uses a small set of those features to make a detection decision [20, $3,2,21]$. Feature re-mapping can be applied with most detectors of this type by simply applying a properly rescaled homography whenever a feature must be looked up, and interpolating as necessary.

We will demonstrate this viewpoint adaptation for two specific pedestrian detectors: the HOG/SVM detector of [20] and the aggregate channel features detector of [2]. These examples illustrate the general approach for both linear and nonlinear classifiers using two popular types of local features, which we will call Haar-like and HOG-like.

The term "Haar-like feature" is used here to describe any local image feature computed as the sum of pixel intensities over a rectangular image region (a "cell"). It may also apply to sums of any pixel-wise function of the image intensity (e.g. sums of squared pixel intensities). These cells are frequently computed in a grid covering the image, as in Figure 3 (left). Each cell has an "extent" $d_{k}=\left[\boldsymbol{u}_{\mathrm{NE}}^{T} \boldsymbol{u}_{\mathrm{SE}}^{T} \boldsymbol{u}_{\mathrm{SW}}^{T} \boldsymbol{u}_{\mathrm{NW}}^{T}\right]$ defined by the coordinates of its corners. To compute the Haar-like feature from a cell in the target viewpoint given the Haar-like features computed from the source (trained) viewpoint, the target-cell extent is transformed via $d_{k^{\prime}}=H_{s t} d_{k}$ using the appropriate source-target homography $H_{s t}$. This extent is then overlaid on the computed (source-view) cell grid, and the result is the sum of the values of the overlapped cells, weighted by the degree to which they overlap the projected extent. Thus the contribution from source-view cell $l$ to target-view cell $k$ is given by

$$
S_{k l}=\frac{\operatorname{area}\left(d_{l} \cap d_{k^{\prime}}\right)}{\operatorname{area}\left(d_{l}\right)}
$$

This is illustrated in Figure 3 (left).

HOG-like features present a slightly greater challenge. They are constructed by computing the gradient (magnitude and angle) across the image, and computing the 9-bin magnitude-weighted angle histograms within each cell. HOG features for a simple image are shown in Figure 4b. HOG gradient angle bins are restricted to a $180^{\circ}$ arc, so that parallel edges are considered to have the same gradient angle and all gradient magnitudes are positive.

HOG feature re-mapping proceeds in two steps: 1) re-mapping the cell locations like for Haar-like features, and 2) re-mapping the angle histogram bins. The second step requires that we infer an angle transformation from the homography $H_{s t}$, and perform a linear resampling over the angle histogram bins similar to what we did for Haar-like features.

Given a homography matrix of the form

$$
H_{s t}=\left[\begin{array}{ccc}
h_{1} & h_{2} & h_{3} \\
h_{4} & h_{5} & h_{6} \\
h_{7} & h_{8} & 1
\end{array}\right],
$$




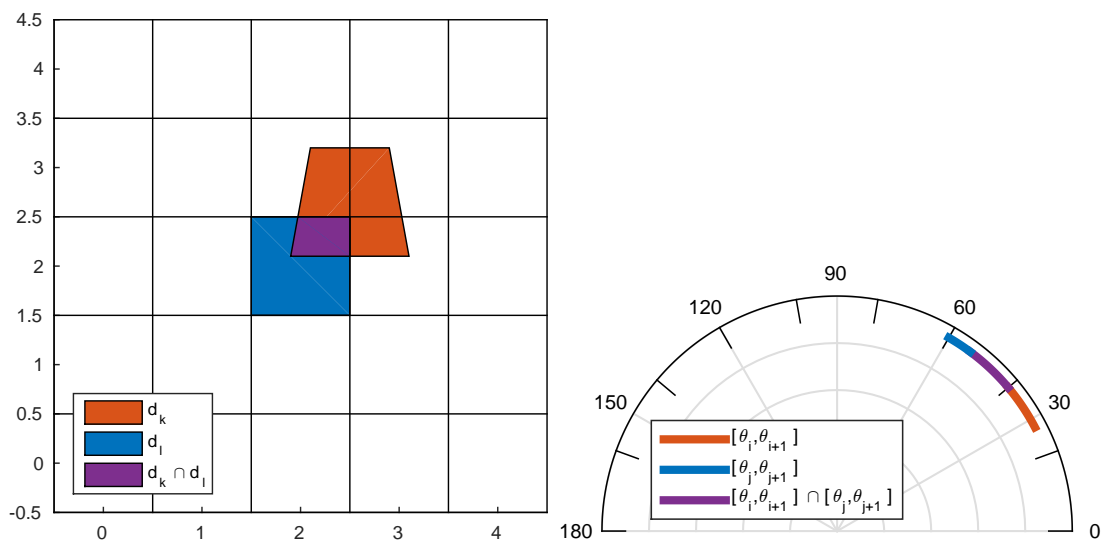

Fig. 3. Left, each cell $d_{l}$ from the first viewpoint is projected to the corresponding location $d_{k}$ in the second viewpoint. Right, each angle histogram bin $\left[\theta_{i}, \theta_{i+1}\right]$ from the first viewpoint is projected to the corresponding location $\left[\theta_{j}, \theta_{j+1}\right]$ in the second viewpoint. The relative overlap of these elements is used to generate a linear feature space mapping.

the gradient angle at a point $\left[x^{\prime}, y^{\prime}\right]$ in the target viewpoint is given by

$$
\theta^{\prime}=\tan ^{-1}\left[\frac{\left(h_{4}-h_{7} y^{\prime}\right) \cos (\theta)+\left(h_{5}-h_{8} y^{\prime}\right) \sin (\theta)}{\left(h_{1}-h_{7} x^{\prime}\right) \cos (\theta)+\left(h_{2}-h_{8} x^{\prime}\right) \sin (\theta)}\right]
$$

where $\theta$ is the corresponding gradient angle in the source viewpoint. This expression is used to map each angle histogram bin edge into the target domain. Then the contribution of computed bin $j$ to desired bin $i$ is given by

$$
A_{i j}=\frac{\operatorname{area}\left(\left[\theta_{j}, \theta_{j+1}\right] \cap\left[\theta_{i^{\prime}}, \theta_{i^{\prime}+1}\right]\right)}{\operatorname{area}\left(\left[\theta_{j}, \theta_{j+1}\right]\right)}
$$

This is illustrated in Figure 3 (right).

Combining cell resampling and histogram resampling, a HOG feature vector $\boldsymbol{x}^{\prime}$ can be warped into a new viewpoint domain according to

$$
\boldsymbol{x}^{\prime} \approx(S \otimes A) \boldsymbol{x}=G \boldsymbol{x},
$$

where $S$ performs the spatial resampling of the HOG cells and $A$ performs the resampling of the angle histogram bins. $\otimes$ denotes the Kronecker product. The two-step HOG feature re-mapping process is illustrated in Figure 4 (a-e). We can see that the proposed feature-remapping algorithm approximates the result of applying an inverse perspective mapping to the image before feature computation.

These feature re-mapping methods for Haar-like and HOG-like features cover a wide variety of the feature types used in modern object detectors. The classification approaches used with these features are arguably more diverse. We 


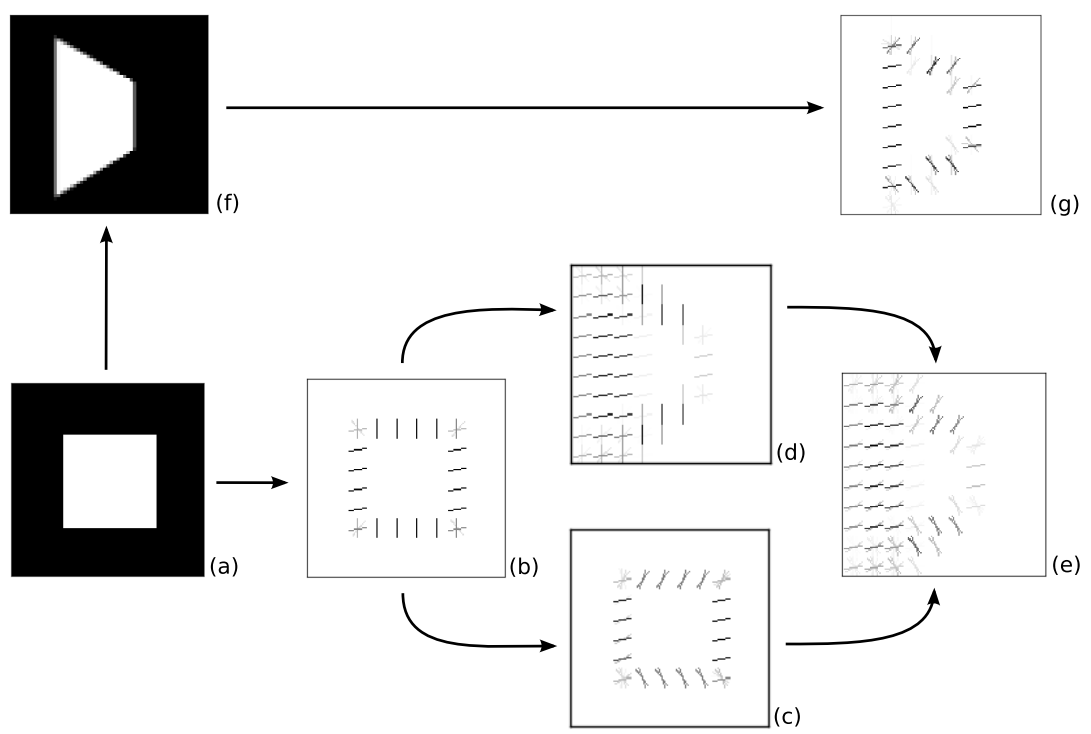

Fig. 4. (a) Example image; (b) HOG features from image; (c) HOG features with histograms resampled; (d) HOG features with cells resampled; (e) HOG features with both histograms and cells resampled; (f) image warped; (g) HOG features from warped image. (e) provides an approximation to $(\mathrm{g})$ without requiring the construction of (f).

focus here on two specific detectors whose modes of operation represent distinct, important classes of detectors.

\subsection{Linear Classifier Adaptation}

The classic HOG/SVM detector learns a linear support vector machine classifier on HOG features [3]. An important aspect of the proposed adaptation procedure is that it does not depend on the method used to train a classifier, but only on the final trained form. Therefore our adaptation of the linear SVM detector is reflective of the procedure for any linear classifier.

The described feature re-mapping method can be used to compute the features for each candidate detection window at run-time, incurring a run-time computational burden. It is possible, however, to achieve exactly the same effect by instead transforming the weights of the trained linear classifier.

The learned classifier weights $w$ and the class label $y$ are defined such that

$$
\begin{aligned}
\boldsymbol{y} & =\boldsymbol{w}^{T} \boldsymbol{x} \\
& =\boldsymbol{w}^{T} G \boldsymbol{x}^{\prime} \\
& =\left(G^{T} \boldsymbol{w}\right)^{T} \boldsymbol{x}^{\prime} .
\end{aligned}
$$


Thus we see that identical results can be obtained by either transforming the testing data to match the source viewpoint or by transforming the classifier weights to match the target viewpoint. This novel result allows the application of a feature space transformation off-line, incurring no run-time computational burden. This viewpoint adaptation method for linear classifiers with Haar-like and HOG-like features has been evaluated for person detections under varying viewpoints using both real and synthetic imagery.

\section{Synthetic Experiments}

The described person detector algorithm is designed to correctly identify persons in images across a wide variety of camera viewpoints as long as training images from one viewpoint are available and the camera pose parameters are known. The evaluation of this detector thus requires an image dataset that contains person images at a wide range of relative perspectives along with corresponding camera calibration information. Though many person image datasets already exist, these properties are not found together in any dataset of significant size. Existing datasets lack viewpoint diversity: Caltech [4], Daimler [22], ETH [23], and TUD-Brussels [24]); camera information: INRIA [3]; and/or person/background diversity: CAVIAR [25] and PETS 2007 [5]. Leveraging 3D modelling software can provide fine control of position and orientation while allowing production of realistic images with a variety of persons and scenes.

\subsection{Multi-view Dataset}

The SketchUp 3D modelling software [26] was used to generate a person detection dataset for the evaluation of viewpoint-informed detection algorithms. A number of synthetic 3D person models and 3D scenes were obtained from the SketchUp 3 D Warehouse [27]. Persons were placed randomly in scenes, rotated about the vertical axis, and exposed to various lighting conditions. Figure 5 shows a subset of the diverse persons and scenes, with random lighting and rotation. The camera was positioned so that it pointed at the person, but at elevations of $0, \pi / 8$, and $\pi / 4$ radians, as illustrated in Figure 2.

The dataset consists of 3 instances $\times 68$ persons $\times 13$ scenes $\times 3$ camera elevations $=7956$ images. These images were cropped and scaled to $64 \mathrm{x} 128$, to be consistent with the INRIA Person Dataset [3]. Background (person-less) images were obtained from 3D scene models without persons present.

\subsection{Performance Results}

A separate linear SVM was trained on HOG features from each of the viewpoints, and each trained classifier was tested on the $\pi / 4$ viewpoint. Then each classifier was adapted to the $\pi / 4$ viewpoint according to the procedure described above, and re-tested. Note that the transformation from the $\pi / 4$ training data to the $\pi / 4$ viewpoint is the identity, so no comparison is necessary. We also reproduced the 

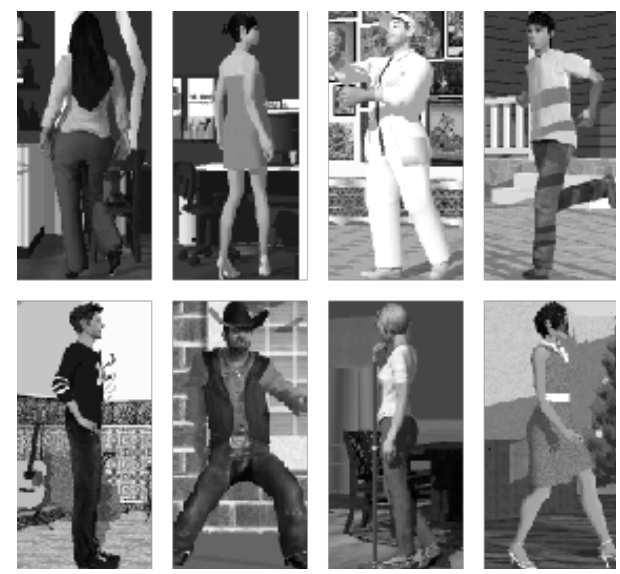

Fig. 5. Some example person images generated using SketchUp. The person models are placed randomly in the scene with random rotation about their vertical axis.

results of [15] using these data by warping each testing image prior to feature extraction. We used nine-fold cross-validation and ensured that neither scenes nor persons were shared between the training and testing sets.

Figure 6 illustrates the performance of each of these detectors. The similarity between the image warping method of [15] and our analogous classifier adaptation is evidence that the HOG feature warping method described above accurately approximates the more computationally expensive image warping. Furthermore, each viewpoint-adapted classifier performs at least as well as the corresponding original classifier, with greater performance gains for source viewpoints farther from the target viewpoint. The main benefit of the proposed approach over that of [15] is in the vastly improved computation time, as we will see in Section ??,.

One potential failing of the proposed viewpoint adaptation algorithm is its reliance on camera pose information. Such information can be acquired by calibration with a known physical target or utilizing some known scene structure [28]. These methods will lead to varying degrees of inaccuracy in the estimated camera pose. We sought, therefore, to explore the robustness of our algorithm with respect to mis-estimation of the camera's elevation.

We used a trained 0-elevation classifier and attempted to apply it to images from an elevation of $\pi / 4$ radians. To model our uncertainty in that testing elevation, we adapted the classifier to a range of elevations from 0 to $\pi / 2$, applying each to the $\pi / 4$ data. The results are illustrated in Figure 7 .

The best performance, measured by the miss rate at $10^{-2}$ false positives per window (fppw), is achieved when the estimated and true target elevation are approximately equal. Performance decays as the estimated elevation decreases toward 0 (the source elevation). As the estimated elevation increases beyond $\pi / 4$, 


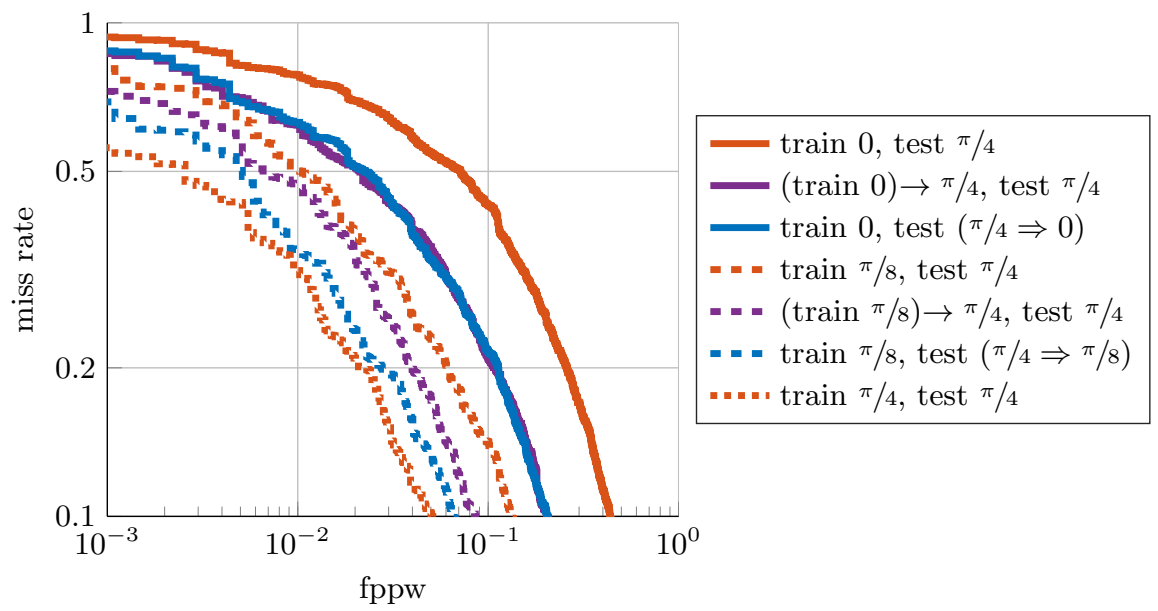

Fig. 6. Performance is shown for a single-view person detector trained on images from three elevations $(0, \pi / 8$, and $\pi / 4)$ and tested on images from $\pi / 4$ elevation (indicated by solid, dashed, and dotted red curves, respectively). Our viewpoint adaptation method is illustrated by the purple curves for each of the mismatched detectors, while the image warping method of [15] is represented by blue curves. The achievable frame rates of these methods, where our approach has the clear advantage, is discussed in Section ??.

performance decays rapidly, falling below the performance of the original, unadapted detector around 1.1 radians. This means that, for this scenario, classifier adaptation is advantageous for errors of up to 0.31 radians $\left(\approx 18^{\circ}\right)$ from the true target elevation. This is much greater than the orientation estimation errors of modern camera calibration approaches, which are on the order of $0.2-0.25^{\circ}[28$, 29].

\section{Nonlinear Classifier Adaptation}

Having seen that the proposed feature/classifier warping method is applicable to Haar-like and HOG-like features with a linear classifier, we would now like to explore the application of this idea to a more powerful modern detector: the ACF pedestrian detector of [2]. As mentioned previously, our adaptation procedure does not depend on how a classifier is trained, but only on how it operates at run-time. Running ACF amounts to evaluating a large set of decision trees, assigning a weight to each outcome, and summing. Each branch of the decision trees implements a threshold on the value of a single feature coming from a specific cell (spatial location) and a specific channel.

This classifier has nonlinear behavior globally, but each individual branching can be considered a simple linear classifier whose weight vector has exactly one non-zero element. Thus our linear classifier adaptation algorithm can be applied 


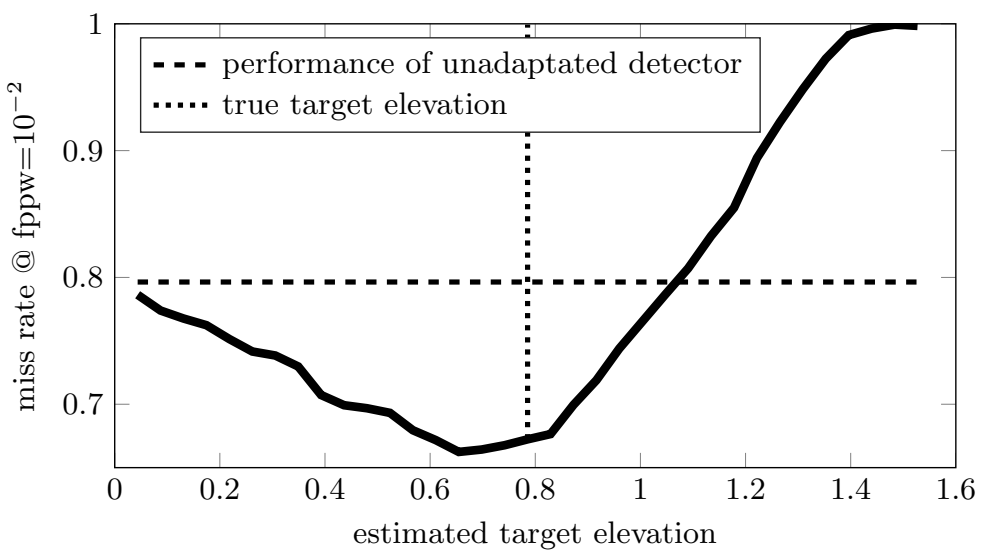

Fig. 7. A trained 0 -elevation detector was adapted to a range of elevations from 0 to $\pi / 2$ radians. It was then evaluated on data from an elevation of $\pi / 4$. The miss rate at $10^{-2}$ false positives per window is plotted as a function of the elevation to which the detector was adapted. Our approach uniformly improves performance until the estimated target elevation is more than 0.31 radians greater than the true elevation.

to each of these weak classifiers, converting the single-feature lookup into a linear combination of a small number of features. In practice, taking just the single bestmatching feature rather than the weighted sum yields nearly equivalent results, so this simplified approach is taken in subsequent evaluations for computational convenience.

We will demonstrate this feature re-mapping approach for ACF with two real pedestrian detection datasets. In shifting from the window classification approach we took with the synthetic data to a realistic object detection framework, we see a secondary benefit of camera pose information: we define candidate detection windows in terms of the $3 \mathrm{D}$ world space rather than the $2 \mathrm{D}$ image space.

\section{Detection Window Selection}

Human detection by searching 3D space was first discussed in [15], and the same principles apply in this work. Essentially, many candidate detection windows in a traditional sliding window detector turn out to be inconsistent with the camera pose (e.g. representing persons standing in the sky).

Building a set of candidate world positions amounts to defining a minimum distance between detections (the detector "stride") and applying the camera projection. The role of the image-space detector stride, measured in pixels, is replaced by a real-world stride, measured in meters. This is illustrated well in Figure 3 of [15]. We can compute the desired real-world stride by analogy with the pixel-space stride. Specifically, ACF assumes that for a any given image 
scale, persons are 96 pixels tall. If persons average 1.75 meters in the real world, then we assume $1.75 / 96 \approx 0.018$ meters per pixel. For a desired pixel stride of 8 , we then have a real-world stride of $8 \times 1.75 / 96=0.146$ meters. Using a fixed real-world stride would yield an infinite number of candidate detection windows in scenes containing the horizon, so we further restrict the detection windows to be no closer than 1 pixel apart in the image space.

It should be noted that each position in the image corresponds to a unique relative viewpoint and associated feature mapping. Thus the detection system must, in general, apply different detection parameters for each image position. These can be computed off-line, however, and simply looked up at run-time.

\section{$7 \quad$ Real Data Results}

The viewpoint adaptation algorithms presented here were applied to real images from two datasets. The PETS 2007 dataset [5] (camera 3) contains camera calibration information, but ground truth was estimated manually using the vbb labeling software [4]. The CAVIAR dataset [25] (Meet_Split_3rdGuy) comes with ground truth, but the camera was calibrated manually using the provided pixel correspondences. The results presented in Figure 8 compare our viewpoint adaptation method for HOG/SVM and ACF to the original, unadapted detectors and to the corresponding image-warping methods [15]. All detectors were trained with the INRIA training data [3].

For the CAVIAR dataset [25], which has some extreme perspective warping effects, all perspective compensation methods improve substantially over the näive detectors. The sharp decrease in miss rate for some detectors around 1 false positive per image can be attributed to a shelf unit visible in the scene. This specific false alarm can also be seen to cause problems in Figure 9 of [15]. The large impact of a single persistent false alarm highlights an issue with using stationary cameras for evaluation of person detection algorithms: we are essentially given a single source of potential false alarms, whose impact is multiplied by the number of frames in the video. Unfortunately, this issue is unavoidable using existing person detection datasets (see Section 4).

For the PETS 2007 dataset [5], which has more moderate perspective effects, the warping methods for the HOG detector provide similar performance benefits, but attempting to adapt the ACF detector actually degrades performance.

This unintuitive result requires some explanation. The standard ACF detector achieves excellent detection performance on most pedestrian detection datasets by training a relatively precise detector and applying it at a large number of positions and scales. Contrast this with the HOG detector, which typically uses a larger cell size to achieve a detector more robust to small changes in the position and scale of detection windows. When these two detectors are applied to the same set of manually extracted detection windows in the image warping scenario, HOG is more robust to sources of error including mis-estimation of the camera pose and persons of different-than-average height. These sources of error outweigh the benefits from inverse perspective warping in the PETS 

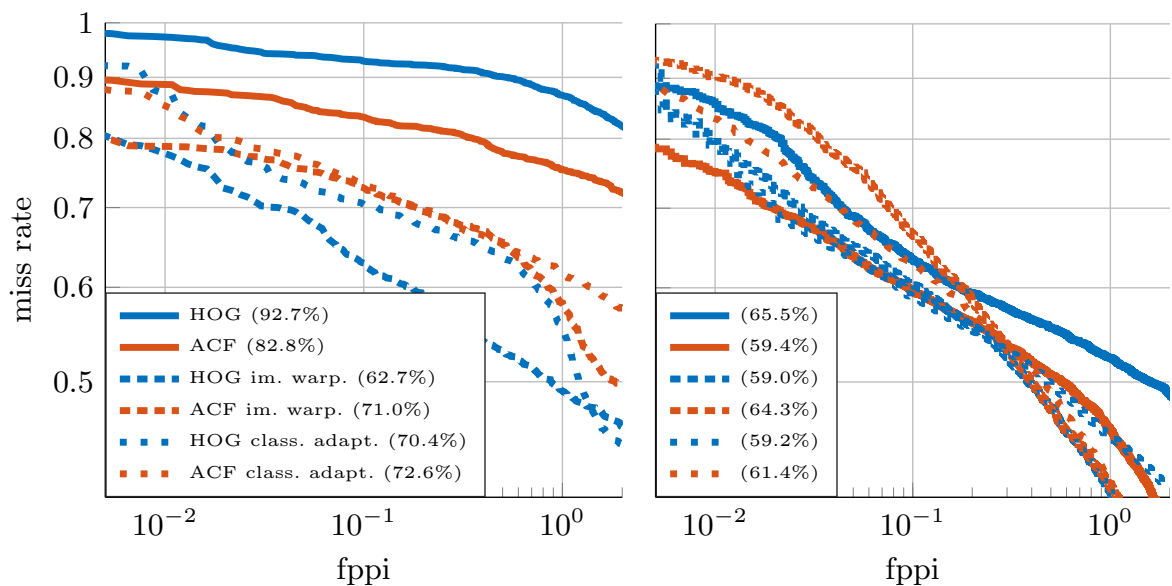

Fig. 8. Performance on images from the CAVIAR dataset (left) and PETS 2007 dataset (right) is shown for the HOG/SVM detector (blue) and the ACF detector (red). The baseline detectors are shown as solid curves, the image-warping method of [15] is shown as dashed curves, and our classifier adaptation methods are shown as dotted curves. The legend indicates the log-average miss rate between fppi of $10^{-2}$ and $10^{0}$ for each detector.

2007 dataset, so we see image warping actually hurt ACF performance. In the CAVIAR dataset, on the other hand, the perspective effects are severe enough that image warping improves the performance of both detectors.

\subsection{Runtime Analysis}

In analysing the runtime of perspective warping methods, it is important to understand that runtime does not depend only on the frame size (as in most such analyses, e.g. [2]), but also on the perspective of the scene. We consider not the number of potential detection windows, but the number of potential 3D positions that persons could occupy in the scene. Clearly, this varies depending on the camera perspective. Thus our analysis looks specifically at the CAVIAR dataset and its associated camera perspective.

Table 1 shows the frame rates (using a single Intel Core i7 CPU) for each method when applied to images from the CAVIAR dataset. The most important observation to make here is that image warping methods operate at more than 15 seconds per frame, while the classifier adaptation methods are even faster than the unmodified detectors. Image warping methods are very slow because they must compute features for each detection window independently. For the CAVIAR dataset, we considered 31603 detection windows, each of which is $128 \times$ 64 pixels, so features must be computed over a total of $2.59 \times 10^{8}$ pixels. For comparison, the unmodified ACF detector, applied to the same image, computes 


\begin{tabular}{|l|l|}
\hline Method & Frame rate (fps) \\
\hline \hline HOG & 0.439 \\
ACF & 3.54 \\
\hline HOG im. warp. & 0.0666 \\
ACF im. warp. & 0.0419 \\
\hline HOG class. adapt. & 11.8 \\
ACF class. adapt. & 10.7 \\
\hline
\end{tabular}

Table 1. Frame rates for each algorithm for the CAVIAR dataset.

features over only $2.36 \times 10^{6}$ pixels. Indeed, we see about 2 orders of magnitude difference in the resulting frame rates.

A couple other observations can be made about these timing results. First, since the computation time for the viewpoint-informed methods is driven by the feature computation time per pixel (without scaling), the adapted HOG detectors are slightly faster than the corresponding adapted ACF detectors, defying the trend in their unadapted counterparts. Second, to ensure a fair comparison to the adapted detectors, the unadapted detectors upsampled the original $384 \times 288$ images by a factor of 4 . This enabled them to detect small-scale persons but also resulted in frame rates somewhat lower than those advertised in the literature for $640 \times 480$ images [2].

The viewpoint-adapted detectors achieve higher frame rates than the unadapted detectors by implicitly using camera pose information to limit the scalepositions at which persons may be detected. The choice of candidate detection windows is discussed in detail in Section 6. This speedup is conceptually similar to that achieved by [19] by using a stereo camera.

\section{Conclusion}

This work is the first to offer a viewpoint adaptation framework limiting the detection search space and handling perspective warping, while retaining frame rates suitable for real-time applications. Our results demonstrate that viewpointadapted detectors can outperform even state-of-the-art detectors that lack perspective correction. Our novel detector adaptation method was demonstrated for linear classifiers, using HOG/SVM as a canonical example, and for the more sophisticated ACF detector [2]. Similar derivations may enable viewpoint adaptation of even more diverse object detection schemes like deformable-parts models [30] and convolutional neural networks [21]. Such implementations are left as future work.

Our viewpoint adaptation algorithm improves detection performance using no training data from the target viewpoint, requiring only information about the pose of the camera with respect the ground plane. Furthermore, the detector adaptation algorithm is fairly robust with respect to mis-estimation of the target viewpoint, providing performance improvements even for relatively large errors 
in the estimated viewpoint. These methods may be extended to explicitly handle uncertainty in the camera pose estimate, as well as to detect objects with more complex 3D models. 


\section{References}

1. Benenson, R., Mathias, M., Tuytelaars, T., Van Gool, L.: Seeking the strongest rigid detector. In: CVPR. (2013)

2. Dollár, P., Appel, R., Belongie, S., Perona, P.: Fast feature pyramids for object detection. PAMI (2014)

3. Dalal, N., Triggs, B.: Histograms of oriented gradients for human detection. In: CVPR. Volume 1. (2005)

4. Dollár, P., Wojek, C., Schiele, B., Perona, P.: Pedestrian detection: An evaluation of the state of the art. PAMI 34 (2012)

5. PETS: Performance evaluation of tracking and surveillance dataset. http://www.cvg.reading.ac.uk/PETS2007/ (2007)

6. Gopalan, R., Li, R., Chellappa, R.: Domain adaptation for object recognition: An unsupervised approach. In: ICCV. (2011)

7. Lowe, D.G.: Object recognition from local scale-invariant features. In: ICCV. Volume 2. (1999)

8. Danielsson, O., Carlsson, S.: Projectable classifiers for multi-view object class recognition. In: ICCV Workshops. (2011)

9. Savarese, S., Fei-Fei, L.: View synthesis for recognizing unseen poses of object classes. In: ECCV. (2008)

10. Thomas, A., Ferrar, V., Leibe, B., Tuytelaars, T., Schiel, B., Van Gool, L.: Towards multi-view object class detection. In: CVPR. Volume 2. (2006)

11. Howe, N.R., Rath, T.M., Manmatha, R.: Boosted decision trees for word recognition in handwritten document retrieval. In: Proceedings of the 28th annual international ACM SIGIR conference on Research and development in information retrieval. (2005)

12. Miyao, H., Maruyama, M.: Virtual example synthesis based on PCA for off-line handwritten character recognition. In: Document Analysis Systems VII. (2006)

13. Tan, X., Chen, S., Zhou, Z.H., Zhang, F.: Face recognition from a single image per person: A survey. Pattern Recognition 39(9) (2006)

14. Kalal, Z., Mikolajczyk, K., Matas, J.: Tracking-learning-detection. PAMI 34(7) (2012)

15. Li, Y., Wu, B., Nevatia, R.: Human detection by searching in $3 \mathrm{~d}$ space using camera and scene knowledge. In: ICPR. (2008)

16. Cao, X., Wang, Z., Yan, P., Li, X.: Transfer learning for pedestrian detection. Neurocomputing 100 (2013)

17. Dai, W., Yang, Q., Xue, G.R., Yu, Y.: Boosting for transfer learning. In: ICML. (2007)

18. Kulis, B., Saenko, K., Darrell, T.: What you saw is not what you get: Domain adaptation using asymmetric kernel transforms. In: CVPR. (2011)

19. Benenson, R., Mathias, M., Timofte, R., Van Gool, L.: Pedestrian detection at 100 frames per second. In: CVPR. (2012)

20. Viola, P., Jones, M.: Rapid object detection using a boosted cascade of simple features. In: CVPR. Volume 1. (2001)

21. Krizhevsky, A., Sutskever, I., Hinton, G.E.: ImageNet classification with deep convolutional neural networks. In Pereira, F., Burges, C.J.C., Bottou, L., Weinberger, K.Q., eds.: Advances in Neural Information Processing Systems 25. Curran Associates, Inc. (2012) 1097-1105

22. Flohr, F., Gavrila, D.M.: PedCut: an iterative framework for pedestrian segmentation combining shape models and multiple data cues. In: Proc. BMVC. (2013) $66-1$ 
23. Ess, A., Leibe, B., Schindler, K., , van Gool, L.: A mobile vision system for robust multi-person tracking. In: IEEE Conference on Computer Vision and Pattern Recognition (CVPR'08), IEEE Press (June 2008)

24. Wojek, C., Walk, S., Schiele, B.: Multi-cue onboard pedestrian detection. In: IEEE Conference on Computer Vision and Pattern Recognition, 2009. CVPR 2009. (June 2009) 794-801

25. CAVIAR: Context aware vision using image-based active recognition benchmark dataset. http://homepages.inf.ed.ac.uk/rbf/CAVIAR/ (2004)

26. Trimble Navigation: SketchUp Make. http://www.sketchup.com (2014)

27. Trimble Navigation: SketchUp 3D Warehouse. https://3dwarehouse.sketchup.com (2014)

28. Robertson, D., Cipolla, R.: Structure from motion. In Varga, M., ed.: Practical Image Processing and Computer Vision. (2009)

29. Antone, M.E., Teller, S.: Automatic recovery of relative camera rotations for urban scenes. In: CVPR. Volume 2. (2000)

30. Felzenszwalb, P., Girshick, R., McAllester, D., Ramanan, D.: Object detection with discriminatively trained part-based models. IEEE Transactions on Pattern Analysis and Machine Intelligence 32(9) (September 2010) 1627-1645 